\title{
Proteomic analysis of dimorphic transition in the phytopathogenic fungus Ustilago maydis
}

\author{
Maik Böhmer ${ }^{1}$, Thomas Colby ${ }^{1}$, Christian Böhmer ${ }^{2}$, Anne Bräutigam ${ }^{1}$, \\ Jürgen Schmidt ${ }^{1 *}$ and Michael Bölker ${ }^{2}$ \\ ${ }^{1}$ Max-Planck Institute for Plant Breeding Research, Köln, Germany \\ ${ }^{2}$ Department of Biology, Philipps University Marburg, Marburg, Germany
}

\begin{abstract}
In the corn smut fungus Ustilago maydis, the dimorphic transition from budding to filamentous growth is intrinsically associated with the switch from a saprophytic to a pathogenic lifestyle. Both pathogenicity and filament formation are triggered by a heterodimeric homeodomain transcription factor encoded by the $b$ mating type locus. Here, we present a reference map of the proteome of this dimorphic phytopathogenic fungus. Using 2-DE in combination with MALDITOF-MS and ESI-MS/MS, we were able to identify 250 distinct proteins obtained from soluble protein samples. In addition, we determined the abundance of cytosolic proteins in filamentous $U$. maydis cells and compared it with that of budding cells. Filamentous growth was induced by two independent regimes, either by overexpression of the bW2/bE1-heterodimer or by overexpression of the small GTP binding protein Rac1. By comparison of expression profiles, we have identified 13 protein spots that were significantly enhanced during filamentous growth induced by bW2/bE1. Rac1 only up-regulates a subset of four of these protein spots. None of these proteins have previously been associated with filamentous growth. Comparison of Rac1- and bregulated protein sets supports the hypothesis that filament formation during pathogenic development occurs via stimulation of a Rac1-containing signalling module.
\end{abstract} Received: November 13, 2006

\section{Keywords:}

Filamentous growth / Phytopathogenesis / Reference map / Two-dimensional gel electrophoresis / Ustilago maydis

\section{Introduction}

The dimorphic phytopathogenic fungus Ustilago maydis has been established as a valuable model system to study fungal dimorphism and pathogenicity [1-3]. In its haploid stage, the fungus is unicellular and multiplies vegetatively by budding (Fig. 1). Haploid cells of compatible mating type recognize each other by specific lipopeptide pheromones and fuse to form the infectious dikaryon [4]. The mating reaction in-

Correspondence: Dr. Michael Bölker, Department of Biology, Philipps University Marburg, Karl-von-Frisch-Str. 8, 35032 Marburg, Germany

E-mail: boelker@staff.uni-marburg.de

Fax: +49-(6421)-2828971

Abbreviations: MIPS, Munich Center for Protein Sequences; MUMDB, MIPS Ustilago Maydis Database
Accepted: December 4, 2006 duces a morphogenetic switch from budding to filamentous growth (Fig. 1). This transition is coordinately controlled by the homeodomain proteins $\mathrm{bE}$ (ast) and bW(est) which form a heterodimeric transcription factor if they are derived from different $b$ mating-type alleles [5]. Haploid strains, that express both the bW2 and the bE1 allele under control of either the arabinose inducible crg promoter (AB31) or the nitrate inducible nar promoter (AB33) have been constructed [6]. Upon induction of $b$ gene expression by shifting the medium composition, these strains undergo a dimorphic transition from budding to filamentous growth (Fig. 1). Strains AB31 and AB33 have been used to detect numerous genes that are transcriptionally regulated by the heterodimeric bW2/bE1 transcription factor upon transition to filamentous

\footnotetext{
* Additorial corresponding author: Jürgen Schmidt, E-mail: jschmidt@mpiz-koeln.mpg.de
} 


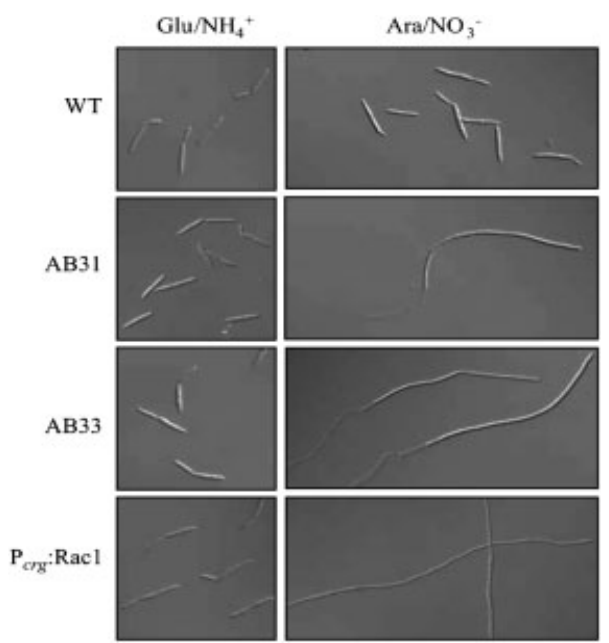

Figure 1. Filament formation in strains $A B 31, A B 33$ and $P_{c r g}:$ Rac1 after shifting cells to inducing medium. Cells were incubated overnight in noninducing medium containing glucose (Glu) and ammonium $\left(\mathrm{NH}_{4}{ }^{+}\right)$and transferred to medium containing arabinose (Ara) and nitrate $\left(\mathrm{NO}_{3}{ }^{-}\right)$. Filament formation was observed after induction of the bW2/bE1 heterodimer by Ara in strain AB31 or by nitrate $\left(\mathrm{NO}_{3}\right)$ in strain $A B 33$, respectively, or after induction of Rac1 in strain FB2- $P_{\text {crg }}$ :Rac1. Wild type cells (FB2) display budding growth under both conditions.

growth using RNA fingerprinting [6] and DNA microarray analysis (J. Kämper, personal communication). Since filament formation is an indispensable part of the pathogenic program, molecular dissection of its temporal and spatial control will help to understand virulence in this model organism.

Small GTP-binding proteins of the highly conserved Rho/Rac family are known to act as molecular switches that control vesicular trafficking, the organization of the actin cytoskeleton and cellular signalling [7]. Molecular characterization of Cdc42 and Rac1 in U. maydis has revealed that these GTP binding proteins regulate distinct morphological changes. While deletion of $c d c 42$ affects cell separation during cytokinesis, deletion of rac1 disturbs cell polarity and abolishes hyphal growth. Remarkably, overexpression of wild type Rac1 induces filament formation in haploid cells ([8]; Fig. 1). Thus, in U. maydis Rac1 is both necessary and sufficient to trigger the dimorphic switch from budding to filamentous growth. In addition, rac1 mutants are nonpathogenic, supporting the central role of filament formation during pathogenic development [8].

The complete genome sequence of $U$. maydis has been determined and its analysis has allowed important insight into the specific adaptations of this fungus to its biotrophic lifestyle [3]. DNA-microarray based studies of genome-wide transcription profiles are extremely useful for selecting candidate genes for functional studies. However, not all changes at the expression level of the encoded proteins are necessarily reflected also at the transcriptional level. It has recently been shown for example, that the b-regulated gene $c l p 1$, which is involved in cell cycle control during hyphal growth, is transcriptionally up-regulated immediately after $b$-locus induction. On the protein level, however, Clp1 is first observed upon plant penetration [9]. Protein accumulation results from the equilibrium between synthesis and degradation and thus posttranscriptional and/or posttranslational processes affect the protein level. The full genome sequence of $U$. maydis has recently been annotated at the Munich Center for Protein Sequences (MIPS) (http://mips.gsf.de/genre/ proj/ustilago/). This information now allows for the first time, large scale proteomic studies of this interesting dimorphic fungal pathogen.

Here, we report the first proteome reference map of U. maydis. More than 250 proteins could be identified combining 2-DE with matrix-assisted laser desorption ionization TOF MS (MALDI-TOF-MS) and ESI-MS/MS analyses. Interactive maps with detailed localization information were generated and are accessible at the MIPS Ustilago Maydis Database (MUMDB; http://mips.gsf.de/genre/proj/ ustilago/Maps/2D/). We have used this reference map to study the changes accompanying the dimorphic transition to filamentous growth at the protein level.

\section{Materials and methods}

\subsection{Strains, plasmids and culture conditions}

$U$. maydis haploid strains FB2 (a2 b2), AB31 (a2 $P_{\text {crg: }}: b W 2$, $b E 1)$ and $A B 33$ ( $\left.a 2 P_{\text {nar }}: b W 2, b E 1\right)$ have been described $[6,10$, 11]. In strain FB2- $P_{\text {crg }}: \operatorname{Rac} 1$ ( $a 2 b 2 P_{\text {crg }}:$ rac 1 ), the endogenous promoter of Rac1 has been replaced by the arabinose-inducible $\mathrm{P}_{\text {crg }}$ promoter [8]. For induction of $b$-locus or rac1 cells were grown overnight in YNB OFF medium $(17 \% \mathrm{w} / \mathrm{v}$ yeast nitrogen base, $0.2 \% \mathrm{w} / \mathrm{v}$ ammonium sulphate, $2 \% \mathrm{w} / \mathrm{v}$ glucose) until $\mathrm{OD}_{600}=0.6-0.8$. Cells were sedimented, washed with YNB ON medium (17\% w/v yeast nitrogen base, $0.2 \%$ $\mathrm{w} / \mathrm{v} \mathrm{KNO}_{3}, 2 \%$ arabinose) and finally resuspended in YNB $\mathrm{ON}$ medium at a density of $\mathrm{OD}_{600}=0.1$. Cells were grown to a final densitiy of $\mathrm{OD}_{600}=1$.

\subsection{Protein preparation}

Cells from a $200 \mathrm{~mL}$ of culture were spun down $(1600 \mathrm{~g}$, $10 \mathrm{~min}, 4^{\circ} \mathrm{C}$ ), washed once in $50 \mathrm{~mL}$ and twice in $25 \mathrm{~mL}$ of extraction buffer (50 mM Tris-Cl, pH 7.5) and finally resuspended in $5 \mathrm{~mL}$ of extraction buffer with $1 \% \mathrm{v} / \mathrm{v}$ protease inhibitor cocktail for yeast (Sigma-Aldrich, Munich, Germany). Cells were frozen in liquid nitrogen and broken using a cell mill (5 min $30 \mathrm{~Hz}$; Retsch, Haan, Germany) in two repetitions with refreezing in liquid nitrogen in between. After centrifugation at $3400 \mathrm{~g}$ for $20 \mathrm{~min}$ at $4^{\circ} \mathrm{C}$, an additional centrifugation step was used to sediment genomic DNA $\left(100000 \mathrm{~g}, 1 \mathrm{~h}, 4^{\circ} \mathrm{C}\right)$ and the supernatant was stored on ice. Protein concentrations were estimated according to Bradford [12] using the dye-binding assay (BioRad, Hercules, CA, USA) 
with BSA as the standard. Protein suspensions were diluted with the three-fold amount of $96 \%$ ethanol and incubated overnight at $-20^{\circ} \mathrm{C}$. After centrifugation at $16000 \times \mathrm{g}$ for 15 min at $4^{\circ} \mathrm{C}$, the pellets were washed twice with $70 \%$ ethanol, dried at room temperature and stored at $-80^{\circ} \mathrm{C}$.

\section{$2.32-\mathrm{DE}$}

2-DE was performed using the NuPAGE ZOOM Benchtop Proteomics system (Invitrogen, Karlsruhe, Germany). Proteins $(100 \mu \mathrm{g})$ were solubilized in $165 \mu \mathrm{L}$ sample rehydration buffer (7 M urea, $2 \mathrm{M}$ thiourea, 2\% Chaps, 0.5\% ZOOM Carrier Ampholytes, pH 3-10 or pH 4-7 (Invitrogen) respectively, $20 \mathrm{mM}$ DTT and $0.1 \%$ bromophenol blue). Prior to IEF ZOOM strips, pH 3-10, NL or pH 4-7 (Invitrogen) respectively were incubated in the rehydration solution containing the sample for $16 \mathrm{~h}$, and IEF was conducted using the following step gradient: 0-175 V (1 $\mathrm{min}), 175 \mathrm{~V}$ (15 min), $175-2000 \mathrm{~V}(45 \mathrm{~min})$ and $2000 \mathrm{~V}(25 \mathrm{~min})$. After IEF, the strips used for gel electrophoresis were first equilibrated in $4.5 \mathrm{~mL}$ lithium dodecyl sulphate (LDS) sample buffer together with $0.5 \mathrm{~mL}$ of $10 \times$ sample reducing agent (Invitrogen), and subsequently in the same solution containing $125 \mathrm{mM}$ iodoacetamide without reducing agent (15 min each). Samples were separated in the second dimension on NuPAGE Novex 4-12\% Bis-Tris ZOOM gels in MES-SDS running buffer (Invitrogen). Proteins were stained with colloidal CBB using Imperial Protein Stain (Pierce, Rockford, IL, USA). Gel images (24-bit.tif) were obtained using a daylight flatbed scanner incorporated in the PROTEINEERsp spot picker system (Bruker, Bremen, Germany). Protein MW and $\mathrm{p} I$ values were estimated from the captured images according to the protein molecular weight markers and selected identified proteins using Proteomweaver 2.2 software (BioRad, Munich, Germany) for calibration.

\subsection{In-gel digestion and MS}

After 2-DE, spots of various intensities were automatically excised and tryptically digested using the DP Chemical 96 Kits for fully automated in-gel digestion (PROTEINEERsp/ $d p$, Bruker). Aliquots of the digests were automatically prepared (PROTEINEERdp, Bruker) for subsequent MALDITOF-MS analysis on AnchorChip targets (Bruker) according to Gobom et al. [13]. Mass spectra of tryptic peptides were taken with a Bruker Reflex IV MALDI-TOF-MS. The obtained PMFs were processed in Xmass 5.1.16 (Bruker) and used to identify the corresponding proteins in the ProteinScape 1.3 database system (Protagen AG, Dortmund, Germany) which triggered MASCOT (Matrix Science, London, UK) and Profound (Genomic Solutions, Huntington, UK) searches against the National Center for Biotechnology Information (NCBI) and MUMDB genome databases. Sample aliquots of spots containing several putative proteins as indicated in Supplementary Table 1 were submitted to indepth LC-MS/MS analyses.

\subsection{LC-MS/MS analysis}

Residual organic solvent was removed from sample digests via vacuum centrifugation prior to injection into a Waters CapLC coupled to a Q-ToFII mass spectrometer (Micromass/ Waters, Milford, MA, USA). Samples were loaded on a Waters Symmetry 300 C18 trapping column for on-column focusing and eluted over a Waters Atlantis C18 analytical column $(75 \mu \mathrm{m} \times 150 \mathrm{~mm})$ with a $5-50 \%$ ACN gradient containing $0.1 \%$ formic acid. The eluant was analysed in survey mode under the control of Masslynx 4.0 (Waters). Multiply charged peaks of sufficient intensity were automatically fragmented in the argon collision cell and the resulting MS/MS spectra were processed in Masslynx 4.0 before submission to MASCOT (Matrix Science) for searches against the NCBI nonredundant database and the MUMDB.

\subsection{Database searching}

PMF data were generated from raw MALDI-MS data using the SNAP algorithm (Bruker) with a signal/noise threshold of 6 and a minimum quality factor of 30 . The PMF data were used to screen the NCBInr database dated 2005/1/25 (http:// www.ncbi.nlm.nih.gov/entrez/query.fcgi?db=Protein) and MUMDB dated 2006/02/22 (http://mips.gsf.de/genre/proj/ Ustilago/) using local MASCOT (MASCOT 2.1.0, Matrix Science). One missed cleavage per peptide was allowed, a mass tolerance of $100 \mathrm{ppm}$ was used for the initial identification. In the cases where no calibrants were identified, the mass tolerance was increased to $200 \mathrm{ppm}$. Carbamidomethylation of cysteine as a fixed modification and oxidation of methionine as a variable modification were taken into account. Mass data collected during ESI-MS/MS analyses were processed and converted into.pkl files. The peak lists were submitted to the MASCOT search engine and searches were performed with a peptide mass tolerance of $0.2 \mathrm{Da}$ and a fragment mass tolerance of $0.1 \mathrm{Da}$. Validation of identifications was performed as described in Section 3.

The MIPS database, together with the TargetP server (http://www.cbs.dtu.dk/services/TargetP/) and the WoLF PSORT server (http://wolfpsort.seq.cbrc.jp) was also used to annotate identied proteins and to assign their predicted subcellular localization. Functional categories were assigned according to the Functional Catalogue Database of MIPS (http://mips.gsf.de/projects/funcat). When multiple functional categories were allotted to an individual protein, the category with the lowest expectation value was chosen.

\subsection{Quantification of protein abundance}

The analysis of protein 2-D images was conducted according to the manufacturer's instructions using Proteomweaver 2.2 software (BioRad, Germany). 2-D images in.tif format were imported into the software and clipping masks for the analysed regions were generated. Spots in the images were then detected and a matchset was created. Spots across all the gel 
images in the matchset were automatically matched and normalized (pair-match based normalization). For all experimental groups, at least two biological replicates were analysed in $\mathrm{pH}$ range $3-10$, one analytical replicate was run in $\mathrm{pH}$ range 4-7. Mean and standard errors were calculated using the software Prism version 4.03 (GraphPad Software, San Diego, USA) based on spot intensities from Proteomweaver 2.2.

To define a threshold for statistically significant changes in protein levels the 100 most intense spot pairs in the two gels representing the saprophytic growth control group were compared with regard to their variation in spot intensity. The SD of the average intensities for this group was $21.52 \%$. The regulation factor between two such groups has a SD of $31.73 \%$. The selected confidence level $(p<0.05)$ results in a trust factor of 1.6. Exponentiation of the SD of the regulation factor with the trust factor results in a minimal significant regulation factor of 1.6. Due to the low sample number, the nonparametric Mann-Whitney-Wilcoxon test has been used to calculate the statistical significance of the differences in spot intensities between different groups. Proteins with a regulation factor above 1.6 and a 0 value in the Mann-Whitney-Wilcoxon test were considered to be significant.

\section{Results}

\subsection{Proteome map of $U$. maydis cytoplasmic proteins}

To facilitate a comparative analysis of expression differences between budding and filamentous cells we first generated a proteome map of $U$. maydis. Total soluble proteins were isolated from budding cells of wild type strain FB2 and from filamentous cells of strains AB31, AB33 [6, 11] and FB2$P_{c r g}$ :Rac1 (a2 b2 $P_{\text {crg }}:$ rac1). All strains are isogenic with the exception of the presence of an active bW2/bE1 heterodimer in strain AB31 and AB33, or the small GTP-binding protein Rac1 in strain FB2- $\mathrm{P}_{\text {crg }}$ :Rac1. Strains were grown in medium containing both arabinose and nitrate. Under these conditions, expression of bW2/bE1 in strains AB31 and AB33, or Rac1 in strain FB2- $P_{\text {crg }}$ :Rac1 is induced. Proteins were separated by 2-D gels using IEF on nonlinear $\mathrm{pH} 3-10$ and linear pH 4-7 strips as first dimension. After IEF, proteins were separated according to their molecular mass in a second dimension and stained with CBB. Using IEF-strips pH 3-10 in the first dimension, up to 382 protein spots could be reproducibly detected, while up to 334 spots were detected using IEF strips $\mathrm{pH} 4-7$. The corresponding protein maps were based on at least two replicate gels. We excised a combined total of 576 spots and merged these into 150 superspots in the $\mathrm{pH}$ range 3-10 and 77 superspots in the $\mathrm{pH}$ range 4-7 using the 2-D gel analysis software Proteomweaver 2.2 (BioRad). Representative 2-D maps of $\mathrm{pH} 3-10$ and $\mathrm{pH} 4-7$ with arbitrary superspot numbers were generated (Fig. 2). Spots were picked from all sections of the gel to ensure equal

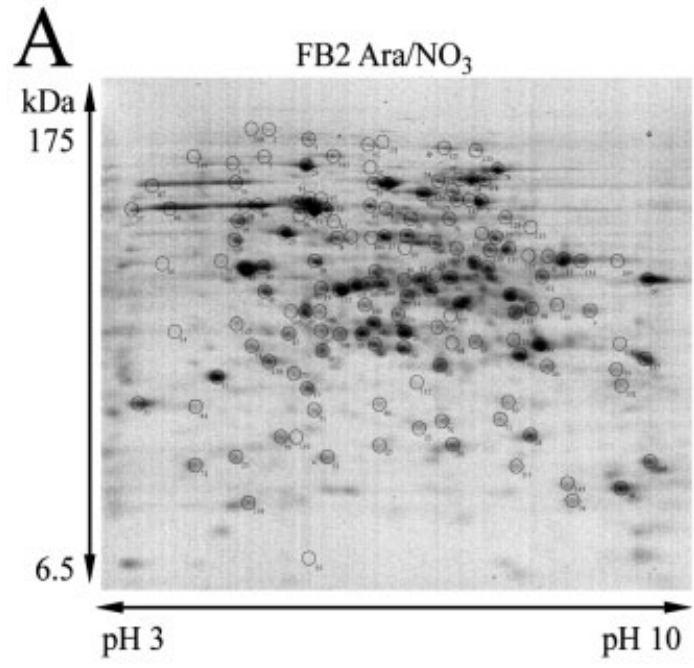

B $\mathrm{FB} 2 \mathrm{Ara} / \mathrm{NO}_{3}$

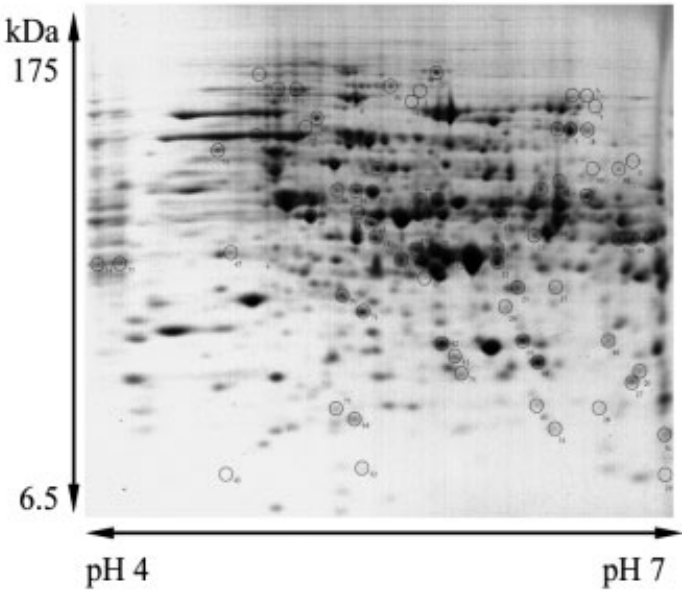

Figure 2. Coomassie-stained 2-D gels of cytosolic protein extracts from $U$. maydis. Proteins were extracted from wild type FB2 cells and from $A B 31, A B 33$ and $P_{\text {crg }}$ :Rac1 cells, all grown under inducing conditions, and separated on 2-D gels. Cytosolic protein extract from wild type FB2 cells were separated by IEF using nonlinear $\mathrm{pH}$ 3-10 (A) and linear $\mathrm{pH}$ 4-7 (B) IEF strips followed by $4-12 \%$ SDS-PAGE. All spots with protein identifications are numbered and are referenced in Supplementary Table 1. Direct accession of 2-D protein information is provided at: http:// mips.gsf.de/genre/proj/ustilago/Maps/2D/.

representation of abundant and less-abundant proteins and to include proteins from a wide range of molecular weights and $\mathrm{p} I \mathrm{~s}$.

\subsection{Protein identification by PMF analysis}

All protein spots were subjected to automated in-gel tryptic digestion and MALDI-TOF-MS analysis (see Section 2) to generate PMFs, which were used to screen the MUMDB and 
the NCBI nonredundant protein database. Throughout our analysis, the MUMDB protein database gave much more reliable identification rates. Protein identifications based on MUMDB were confirmed by searching the PMFs against NCBI database sequences from fungi. The analysis resulted in the identification of 250 distinct proteins (Supplementary Table 1). The reliability of protein identifications was assessed by the following criteria: good agreement of the predicted $\mathrm{p} I$ values and masses with the observed position on the gel, the MASCOT score [14] and the extent to which the observed PMF could be explained by the sequence of the predicted proteins.

The average deviations of the measured peptide masses compared to the theoretical values of the respective peptides of the identified proteins were less than $0.1 \mathrm{Da}$. For the database used (6900 proteins), a MASCOT score or 51 or higher translates to an expectation value of 0.05 or lower [14]. In samples containing mixtures, however, the scores of individual components are systematically lowered since only part of the PMF is explained by the individual proteins. MASCOT scores for individual proteins observed in multiple protein spots therefore often fell below the significance threshold, although the identified proteins satisfied the other criteria. Independent analysis of selected multiprotein samples by ESI-MS/MS, however, confirmed in all cases the proteins identified on the basis of the PMFs.

The identified proteins ranged in calculated molecular masses from 10.3 to $122.8 \mathrm{kDa}$ and in calculated $\mathrm{p}$ Is from 4.2 to 10.3. In two cases, protein spots were identified which contained fragments of polypeptides that have been annotated only as partial protein sequences in the U. maydis database since their ORFs are interrupted by a sequencing gap. In both cases, we could thus confirm that these fragments belong to a single protein. These were an arginyl tRNA synthetase in spot 4 (UM11664 and UM11814) and PGI1 (glucose-6-phosphate isomerase) in spot 69 (UM11748 and UM11045). In both cases, the sum of the predicted fragment masses corresponded to the total mass observed on the 2-D gel.

\subsection{Subcellular localization and functional categories}

Since we used soluble proteins for the analysis, we expected that most of the identified proteins are either localized in the cytoplasm or associated with organelles. To test this hypothesis, we used the annotation of identified proteins to deduce their subcellular localization. In all cases where no such information was given, subcellular localization was predicted by the programs TargetP and WoLF PSORT [15, 16]. As expected, the largest fraction appeared to be cytosolic (41\%), $26 \%$ were predicted to be in mitochondria, while the other proteins were presumably located in both cytosol and nucleus $(12 \%)$, in the nucleus $(8 \%)$, in the secretory compartment $(6 \%)$, in peroxisomes $(4 \%)$, or in the ER $(1 \%)$. Identified proteins $(2 \%)$ were associated with the cytoskeleton. Surprisingly, membrane-bound proteins were also identified. However, most of these proteins were predicted to be localized in one of the mitochondrial membranes (e.g. the subunits of ATPase (UM06324, UM10397, UM10548 and UM05090) and a porin-related protein (UM05662). Other membrane proteins were derived from the vacuolar membrane (e.g. vacuolar ATPase (UM11698, UM10926 and UM11271)) or the peroxisomal membrane (UM10331). No proteins predicted to be integrated in the plasma membrane were identified.

We used the MIPS Functional Categories Database (FunCat) to classify the identified proteins (250) according to function. The majority of polypeptides were classified as being involved in energy (33\%) or metabolism (29\%), whereas $15 \%$ of proteins could not be classified. Other proteins are likely involved in binding or cofactor requirement $(13 \%)$, protein fate $(3 \%)$, protein synthesis $(3 \%)$, biogenesis of cellular components $(2 \%)$, cell rescue, defense and virulence $(2 \%)$, cell cycle and DNA processing $(<1 \%)$ as well as cellular transport $(<1 \%)$. In comparison to the full genome, proteins involved in the generation of energy and metabolic pathways were significantly overrepresented among the proteins identified in the 2-D gel. Only $5 \%$ of all $U$. maydis genes are annotated as being involved in energy generation and $17 \%$ are predicted to encode metabolic enzymes [17]. On the other hand, unclassified proteins (genome: 70\%) were strongly underrepresented in our subset, most likely due to low expression levels.

\subsection{Proteins identified in multiple isoforms}

Many proteins are subject to PTMs such as phosphorylation, acetylation, ubiquitinylation or glycosylation. Proteome analysis allows the detection of such modifications by the appearance of multiple spots associated with a single protein. Although some of these modifications do not significantly change the molecular weight of a protein, they often cause a shift in $\mathrm{pI}$ in either the acidic or basic direction. To test whether such patterns were the result of phosphorylation, we stained 2-D gels of all strains analysed with the phospho-protein specific dye Pro-Q Diamond (Molecular Probes) and analysed individual spots. We identified the ribosomal protein RPS19 (UM11551) in two phosphorylated forms (data not shown). Interestingly, RPS19 appears to be highly phosphorylated since it could not be detected on Coomassie stained gels. In humans, RPS19 has recently been shown to interact with the oncogenic kinase Pim1, which is able to phosphorylate RPS19 in vitro [18].

Another prominent protein, which appeared as a string of three spots ( $\mathrm{pH} 3-10$, spots 44, 105, 106) corresponded to actin (UM11232). However, these protein spots could not be stained with Pro-Q Diamond, indicating that most of the actin in the cell is nonphosphorylated. Further analysis by ESI-MS/MS has shown that actin is N-terminally acetylated without further N-terminal processing as shown for other fungi [19]. 


\subsection{Identification of proteins associated with filamentous growth}

To identify changes in the expression of proteins associated with the dimorphic switch of $U$. maydis, we compared total protein extracts of AB31, AB33, FB2- $\mathrm{P}_{\text {crg }}$ :Rac1 (a2 b2 $P_{\text {crg: }}$ (ac1) and FB2 wild type cells (Fig. 3). All cells were grown in inducing medium containing nitrate as nitrogen source and arabinose as carbon source. Under these conditions only wild type cells (FB2) grow by budding. AB31 and AB33 cells express the heterodimeric bW2/bE1 transcription factor under control of the arabinose-inducible $\mathrm{crg}$ promoter or the nitrate dependent nar promoter, respectively and thus grow as long filaments (Fig. 1). FB2- $\mathrm{P}_{\text {crg }}$ :Rac1 cells also grows as filaments due to overexpression of the small GTP-binding protein Rac1 [8]. In all cases, the morphogenetic switch from budding to filamentous growth was observed around $2 \mathrm{~h}$ after induction, with all cells growing as filaments after at least $5 \mathrm{~h}$. To ensure that the proteome pattern reflects the characteristics of full filamentous growth, cytosolic protein extracts were prepared from cells grown for $24 \mathrm{~h}$ under inducing conditions. Coomassie-stained images of 2-D gels were acquired by scanning and all spots were matched by gel-to-gel comparison, using Proteomweaver 2.2 software, so that differences in the relative intensities of each protein could be analysed. For all samples, at least two biological replicates were analysed. Good reproducibility of spot intensities was obtained among the replicates of each strain. The $\mathrm{SD}$ of the average intensities for gels representing wild type cell extracts was $21.5 \%$. Thus, a regulation factor of 1.6 was calculated as being significant (for details see Section 2).

A total of 13 protein spots showed significant differences between the filamentously growing AB31, AB33 and the budding wild type cells (see Table 1, Fig. 3). Ten of these 13 spots represented single proteins, while the remaining protein spots harbored a mixture of proteins. Among the proteins identified were some whose genes have been previously described as being induced by expression of an active $b$-locus (e.g. protein disulphide isomerase [6]. Others have been identified in a screen for genes regulated by cAMP-signalling (e.g. the aldo-keto reductase YakC, actin [20] and SEP3 [21]. However, additional proteins, not yet detected in other screens were also found to respond to $b$-locus induction. These include glutaminase A, UTP-glucose-1-phosphate uridyltransferase, a predicted 1,4-benzoquinone reductase and homologs of the yeast proteins Cdc48 and Pep4. Most of the b-regulated proteins, except glutaminase A, showed a minor level of basic expression in budding cells, and expression was significantly increased in the filaments. Since in these experiments most filament-associated proteins had $\mathrm{p} I$ values in the range of $\mathrm{pH} 4-7$, IEF strips with a narrower $\mathrm{pH}$ range were used in the first dimension to improve resolution. Spot 18, showing an increase in filamentous growth below threshold, could be separated into two spots $\left(19^{\star}, 73\right)$ which showed a much stronger increase after induction of the $b$-locus. Unfortunately, after comparing the PMF's

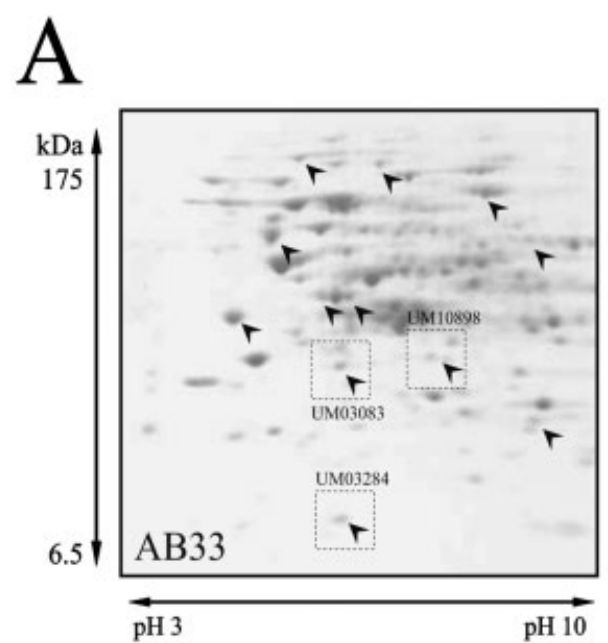

$\mathrm{B}$

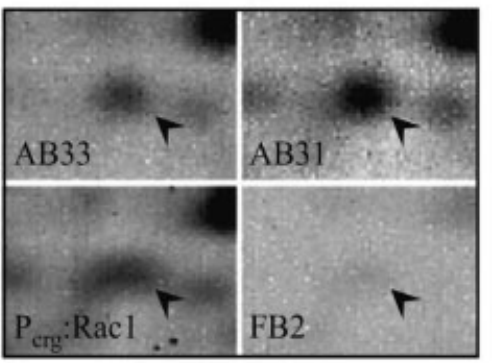

short chain alcohol dehydrogenase (UM10898)

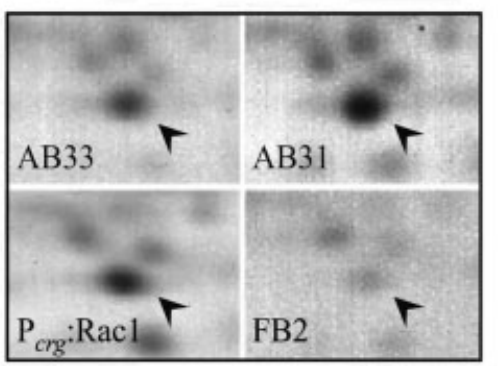

conserved hypothetical protein (UM03083)

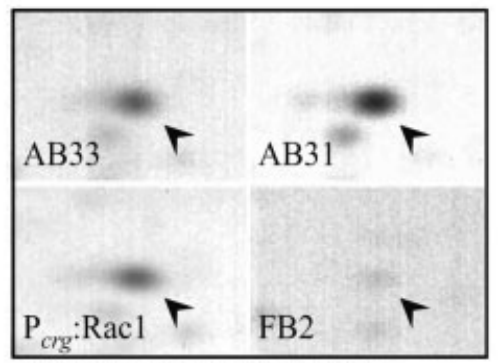

CipC homologue (UM03284)

Figure 3. Up-regulated proteins after induction of filamentous growth by expression of either bW2/bE1 or Rac1. (A) Arrows mark proteins up-regulated in strain $A B 33$ after transition to filamentous growth by $b$-locus induction. (B) Enlargement of some protein spots up-regulated in filaments induced by over-expression of either bW2/bE1 or Rac1. 
Table 1. Protein spots with increased intensities after transition to filament inducing conditions as compared to wild type cells

\begin{tabular}{|c|c|c|c|c|c|c|c|}
\hline \multirow{2}{*}{$\begin{array}{l}\text { Spot } \\
\text { no. }{ }^{\text {al }}\end{array}$} & \multirow{2}{*}{$\begin{array}{l}\text { MUMDB } \\
\text { accession }\end{array}$} & \multirow[t]{2}{*}{ Description } & \multirow{2}{*}{$\begin{array}{l}\text { Subcellular } \\
\text { localization }\end{array}$} & \multirow{2}{*}{$\begin{array}{l}\text { Functional } \\
\text { category }\end{array}$} & \multicolumn{3}{|c|}{ Fold change at a time $e^{\text {b) }}$} \\
\hline & & & & & AB31 & AB33 & Rac1 \\
\hline \multicolumn{8}{|c|}{$b$-Locus and Racl induced } \\
\hline $\begin{array}{l}53 \\
32\end{array}$ & UM03284 & CipC homologue & Nucleus & - & $\begin{array}{l}4.3 \pm 0.1 \\
\text { fs }\end{array}$ & $\begin{array}{l}3.8 \pm 1.2 \\
\text { fs }\end{array}$ & $\begin{array}{l}2.1 \pm 1.2 \\
\text { fs }\end{array}$ \\
\hline 112 & UM10898 & Related to short chain alcohol dehydrogenase & Mitochondria & Metabolism & $2.2 \pm 0.2$ & $2.4 \pm 0.2$ & $2.2 \pm 0.3$ \\
\hline 17 & UM03083 & Related to phosphoserine phosphatase & Cytosol & - & $2.2 \pm 0.1$ & $2.0 \pm 0.1$ & $1.8 \pm 0.0$ \\
\hline \multirow[t]{3}{*}{5} & UM04971 & OSM1 & Mitochondria & Energy & $1.6 \pm 0.1$ & $2.0 \pm 0.5$ & $1.1 \pm 0.1$ \\
\hline & UM04967 & Transketolase & Cytosol & Energy & & & \\
\hline & UM03069 & Acetyl-CoA synthetase & Cytosol & Energy & & & \\
\hline \multirow[t]{3}{*}{8} & UM04971 & OSM1 & Mitochondria & Energy & 4.3 & 4.3 & 3.4 \\
\hline & UM04967 & Transketolase & Cytosol & Energy & & & \\
\hline & UM03069 & Acetyl-CoA synthetase & Cytosol & Energy & & & \\
\hline \multirow[t]{3}{*}{9} & UM04971 & OSM1 & Mitochondria & Energy & 2.2 & 2.1 & 1.6 \\
\hline & UM04967 & Transketolase & Cytosol & Energy & & & \\
\hline & UM03069 & Acetyl-CoA synthetase & Cytosol & Energy & & & \\
\hline \multicolumn{8}{|c|}{$b$-Locus induced } \\
\hline \multirow[t]{2}{*}{10} & UM04971 & OSM1 & Mitochondria & Energy & 3.2 & 2.6 & 1.1 \\
\hline & UM04967 & Transketolase & Cytosol & Energy & & & \\
\hline 92 & UM06158 & Glutaminase A & Secretory pathway & Metabolism & fs & fs & - \\
\hline 3 & & & & & fs & fs & - \\
\hline 14 & UM04926 & PEP4 & Secretory pathway & $\begin{array}{l}\text { Cell rescue, defense } \\
\text { and virulence }\end{array}$ & $2.2 \pm 0.3$ & $3.1 \pm 0.7$ & $1.0 \pm 0.2$ \\
\hline 127 & UM05584 & UTP-glucose-1-phosphate uridyltransferase & Mitochondria & Energy & $2.0 \pm 0.2$ & $2.9 \pm 0.8$ & $1.2 \pm 0.1$ \\
\hline 1 & UM11271 & $\mathrm{CDC} 48$ & Nucleus & Energy & $2.0 \pm 0.1$ & $2.1 \pm 0.1$ & $1.4 \pm 0.0$ \\
\hline 6 & UM10156 & Protein disulphide isomerase & Secretory pathway & $\begin{array}{l}\text { Biogenesis of } \\
\text { cellular components }\end{array}$ & $1.5 \pm 0.2$ & $1.7 \pm 0.3$ & $1.2 \pm 0.3$ \\
\hline 113 & UM10387 & 1,4 Benzoquinone reductase & Cytosol & Metabolism & $1.7 \pm 0.4$ & $1.6 \pm 0.1$ & $1.5 \pm 0.5$ \\
\hline 26 & & & & & 2.3 & 4.8 & 1.3 \\
\hline 27 & & & & & 2.3 & 2.3 & 1.2 \\
\hline \multirow[t]{5}{*}{18} & UM11944 & GCY1 & Cytosol & Energy & $1.5 \pm 0.3$ & $1.5 \pm 0.2$ & $1.0 \pm 0.1$ \\
\hline & UM11098 & GLN1 & Cytosol & Metabolism & & & \\
\hline & UM10681 & ILV5 & Mitochondria & Metabolism & & & \\
\hline & UM03537 & Ornithine carbamoyl transferase & Mitochondria & Metabolism & & & \\
\hline & UM00674 & FBA1 & Cytosol & Energy & & & \\
\hline \multirow[t]{3}{*}{$73^{\ddagger}$} & UM11098 & GLN1 & Cytosol & Metabolism & 2.0 & 1.8 & 1.0 \\
\hline & UM01708 & HOM6 & Secretory pathway & Metabolism & & & \\
\hline & UM00674 & FBA1 & Cytosol & Energy & & & \\
\hline \multirow[t]{4}{*}{19} & UM11098 & GLN1 & Cytosol & Metabolism & 3.9 & 3.1 & 1.5 \\
\hline & UM03537 & Ornithine carbamoyl transferase & Mitochondria & Metabolism & & & \\
\hline & UM03006 & Aldo-keto reductase YakC & Nucleus & Metabolism & & & \\
\hline & UM00674 & FBA1 & Cytosol & Energy & & & \\
\hline 106 & UM11232 & Actin & Cytoskeleton & $\begin{array}{l}\text { Binding } \\
\text { function }\end{array}$ & $2.5 \pm 1.3$ & $1.8 \pm 0.4$ & $1.2 \pm 0.0$ \\
\hline \multirow{2}{*}{105} & UM11232 & Actin & Cytoskeleton & Binding & $1.9 \pm 0.3$ & $1.5 \pm 0.1$ & $1.1 \pm 0.2$ \\
\hline & UM03449 & SEP3 & Mitochondria & $\begin{array}{l}\text { function } \\
\text { Metabolism }\end{array}$ & & & \\
\hline
\end{tabular}

a) Spot numbers as depicted in Supplementary Table 1, Supplementary Table 2 and Fig. $1, *$ indicates spots identified in the pH range 4-7.

b) Fold change at a time = relative fold change versus wild type induced samples. The relative fold changes are averaged values of duplicate experiments, values are given \pm standard error and fs indicates filament specific expression. 


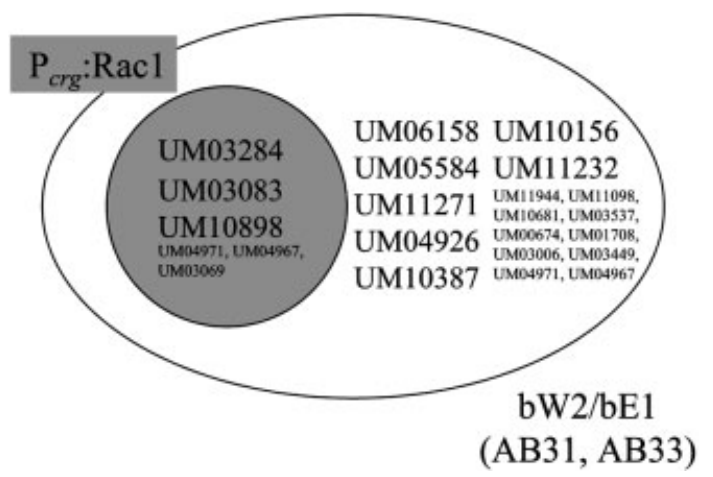

Figure 4. Rac1 induces a subset of b-regulated genes during filament formation. The Venn diagram shows all proteins identified from protein spots that are up-regulated after $b$-locus-induction. Upon induction of Rac1 a subset of three proteins is up-regulated. Accessions in smaller fonts represent proteins from multiple protein spots.

derived from these protein spots obtained under induced and noninduced conditions, it was not possible to allocate individual proteins to these spots, suggesting a mixture of proteins with possible PTMs.

Four of the b-regulated protein spots also appear to be differentially expressed in FB2- $\mathrm{P}_{\text {crg }}$ :Rac1 and wild type cells (see Table 1, Fig. 3). Remarkably, no protein spots could be identified that showed an enhanced intensity exclusively after rac1-induction (Fig. 4). The shared subset of proteins whose expression is induced both either an active bW2/bE1 transcription factor or by overexpression by the Rac1-GTPase contains a CipC homologue, a protein related to short chain alcohol dehydrogenase and a phosphoserine phosphatase. With a narrower $\mathrm{pH}$ range the multiprotein-spot 5 could be separated into three individual spots $\left(8^{*}, 9^{\star}, 10^{*}\right)$. Spot $8^{*}$ showed a 3-4-fold and spot $9 *$ showed a 1.6-2-fold increase after induction of filamentous growth by $b$-locus- and rac1induction. In contrast, spot $10^{*}$ only showed an increase after $b$-locus-induction. Unfortunately, these spots could not be assigned to individual proteins either.

We analysed 13 protein spots that were identified as being up-regulated by bW2/bE1 and/or Rac1 for induction by arabinose or nitrate in the media by comparing wild type cells grown in noninducing and inducing medium. For three protein spots, identified as protein disulphide isomerase, PEP4 and a multiprotein spots consisting of actin and SEP3, a significantly higher expression level was detected in the inducing medium. However, $b$-locus- and rac1-induction still led to even higher protein levels (data not shown).

\section{Discussion}

We have generated the first proteomic reference map of the phytopathogenic fungus $U$. maydis with coverage of $4 \%$ of all annotated genes. Within this subset, we have identified 13 protein spots with a significant increase in intensity after dimorphic transition from budding to filamentous growth induced by expression of either the bW2/bE1 transcription factor or the small GTP-binding protein Rac1. Among these filament-associated proteins three were already known to respond to $b$-locus-induction and thus served as useful internal control to validate the experimental system. Protein disulphide isomerase (UM10156) has been identified previously as b-regulated gene by RNA fingerprinting [6]. The increases in protein amounts of putative glutaminase A (UM06158) and the potential hydrolase UM03083 as measured in this study corresponds well with transcription induction rates determined by genome-wide expression analysis using DNA arrays (J. Kämper, personal communication). Within a 12-h time course UM03083 showed a three-fold induction on transcriptomic level and a 2.0-2.2-fold induction in protein amount. For glutaminase A an 85-fold induction was measured by microarray analysis (J. Kämper, personal communication). The high level of induction may explain the filament specific appearance of this protein in our study. Furthermore, b-regulated genes identified by microarray analysis (J. Kämper, personal communication) are present on our proteome map as well, e.g. versicolorin B synthase (UM03246) and anthranilate synthase (UM02376). However, both proteins are minor components of multiprotein spots. Therefore, the 4- and 13-fold induction observed at the transcriptional level was obviously not sufficient to affect the abundance of the whole protein spot significantly. It cannot be ruled out, however, that the differences between transcriptional induction and changes at the protein level may be at least partially due to the fact that different time points (24-h and $12-\mathrm{h}$ post induction, respectively) were used in these studies.

Approximately $25 \%$ of the proteins we identified to be up-regulated exclusively by the b-heterodimer harbor a secretion signal as predicted by TargetP. This is significantly higher than the $6 \%$ of secreted proteins identified genome-wide [3] and in our proteome map (Supplementary Table 1). The b-regulated secreted proteins include PEP4, glutaminase A, HOM6 and protein disulphide isomerase. Protein disulphide isomerase, which catalyses the proper formation of disulphide bridges may play a role in protein secretion itself, since elevated levels of this enzyme are required in S. cerevisiae for efficient protein secretion [22]. Since secreted proteins would be expected to reside in the culture supernatant we assume that most of the identified proteins carrying a secretion signal are either still in the endoplasmatic compartment or are associated with the cell wall. Interestingly, gene clusters encoding secreted proteins were shown to play a decisive role during pathogenic development in planta [3]. But none of these candidates were identified in our study indicating that the expression of these gene clusters is strictly limited to pathogenic development. 


\subsection{Filament specific proteins}

Filament formation is characterized by highly polarized tip growth and is critical for pathogenic development in U. maydis and other dimorphic pathogenic fungi (reviewed in [23]). Therefore, understanding of the molecular underpinnings of this morphogenetic program is crucial to elucidate the pathogenic development. In $U$. maydis, filament formation is observed after fusion of compatible haploid cells on artificial media that contain charcoal [24]. In this case, aerial hyphae are formed that emerge from fungal colonies. These hyphae are hydrophobic to prevent desiccation of fungal mycelium. A filament-specific repellent protein, Rep1, has been isolated from cell walls of aerial hyphae [25]. Although Rep1 is transcriptionally regulated by the $b$ heterodimer [6], it is processed after secretion into short peptides which are too small in order to be identified in 2-D gels [25].

In our screen, we were able to identify 13 protein spots whose expression was significantly increased in filaments compared to budding cells. Although this number may appear small, these proteins are expressed at a level high enough to be detected on Coomassie-stained 2-D gels. Thus, these proteins are expected to exert important functions during this morphogenetic program.

\subsection{Proteins up-regulated in both $b$ - and Rac1-dependent filaments}

To identify proteins that are intrinsic to filamentous growth independent of its induction, we searched for proteins that are up-regulated upon both bW2/bE1- and Rac1-induced filament formation. It has been suggested that the small GTP-binding protein Rac1 acts as a molecular switch that directly triggers polarized growth at the hyphal tip [8]. Therefore, we reasoned that protein spots up-regulated by either bW2/bE1 or Rac1 correspond to those proteins that are obligate components of filaments in $U$. maydis. Within the small subset of four coregulated protein spots, we identified a homologue of CipC (UM03284), a small conserved fungal protein with unknown biological function. CipC was first identified as a concanamycin induced protein in Aspergillus nidulans and homologues have since been found to be induced under many different conditions: it is up-regulated during ectomycorrhizal symbiosis in Paxillus species, in experimental cryptococcal meningitis and by nitrogen starvation in Gibberella fujikuroi [26-29]. CipC is also significantly up-regulated under conditions of nitrogen starvation in U. maydis (M. Bölker, unpublished results). Nitrogen starvation has been shown to be one of the environmental cues for disease development in planta in a number of different fungi (reviewed in [30]). Multiple genes are induced both by nitrogen starvation and in planta, as shown in Cladosporium fulvum [31], Magnaporthe grisea [32] and Colletotrichum gloeosporioides [33]. A direct link between nitrogen starvation and morphology exists in S. cerevisiae, where nitrogen star- vation causes a pseudohyphal, invasive growth pattern [34]. The importance of CipC for filament formation and nitrogen starvation will be studied further by mutational analysis.

The second protein associated with filamentous growth was a conserved protein, UM03083, which contains a hydrolase domain and shows similarities to phosphoserine phosphatases. This might indicate that dephosphorylation of specific target proteins is crucial for filament formation. In addition, a short chain alcohol dehydrogenase (UM10898) was also induced in cells growing as filaments. The enhanced level of this putative enzyme might either reflect a different metabolism during filamentation or be involved in the production of certain components required for hyphal cell wall synthesis.

Interestingly, all proteins up-regulated by Rac1 were also found to be induced by $b$-locus and no protein could be identified in our study that was only regulated by Rac1 (Fig. 4). This supports the notion [8] that Rac1-activity is stimulated at the growing tip by the $b$-locus and then directly triggers filament formation. This hypothesis is supported by the observation that Rac1 can induce filament formation in the absence of an active bW/bE transcription factor but an active $b$-locus can not induce filament formation in rac1mutants [8]. Stimulation of Rac1-activity during b-induced filament formation must occur by specific activation since the low level of induction at the transcriptional level (twofold) is certainly not sufficient to trigger filament formation [8]. Taken together, our data demonstrate that Rac1 is both necessary and sufficient to trigger filament formation. During pathogenic growth its activity is stimulated by the $b$-locus presumably by a Rac1-specific guanine nucleotide exchange factor.

\subsection{Interplay of signalling pathways involved in filament formation}

Mutants defective in cAMP signalling display constitutive elongated cell morphology [35-37]. By subtractive hybridization 26 genes were identified that were specifically expressed in U. maydis uac1 mutants, which lack adenylyl cyclase [20]. Among these genes were rep1, actin and a homologue of the YakC kinase [20]. Two genes encoding catalytic subunits of protein kinase A ( $a d r 1$ and $u k a 1)$ have also been identified as part of cAMP signalling [38, 39]. In the U. maydis adr1 mutant strain the septin SEP3 has been identified as being up-regulated in these filamentously growing cells [21]. The cAMP pathway thus suppresses expression of these genes. Later in the infection processes repression of these genes may be suppressed by transcription factors, such as the Sql1Tub1 complex [40].

In our study, actin, YakC and SEP3 were identified as proteins up-regulated by the $b$-locus but not by Rac1 (Table 1). This may indicate that an active $b$ heterodimer can suppress cAMP signalling and thereby induce these genes. Potential links between cAMP signalling an b-signalling could be the MAPK cascade that acts in parallel to the cAMP 
pathway [41] or the pheromone response system that is repressed after activation of the $b$-locus [42].

Repression of the cAMP/PKA signalling pathway therefore seems to be controlled by the $b$-locus and to act in parallel to Rac1 signalling, but influences a different set of genes (Fig. 5). Evidence for a potential role of this subset comes from a recent analysis, which showed that the $U$. maydis septin SEP3 is not specifically required for filament formation but is critical for the morphology of both budding and filamentous cells [36]. Septins are GTP-binding proteins with a distinct role during cellular division and morphology [43]. This may explain why the generation of mating filaments and filamentous growth in planta is not impaired in sep3 mutants but that an altered morphology is observed [36].

The cAMP signalling pathway plays a crucial role during morphogenesis and pathogenic development [44], and there is intensive crosstalk between b-signalling, the pheromone

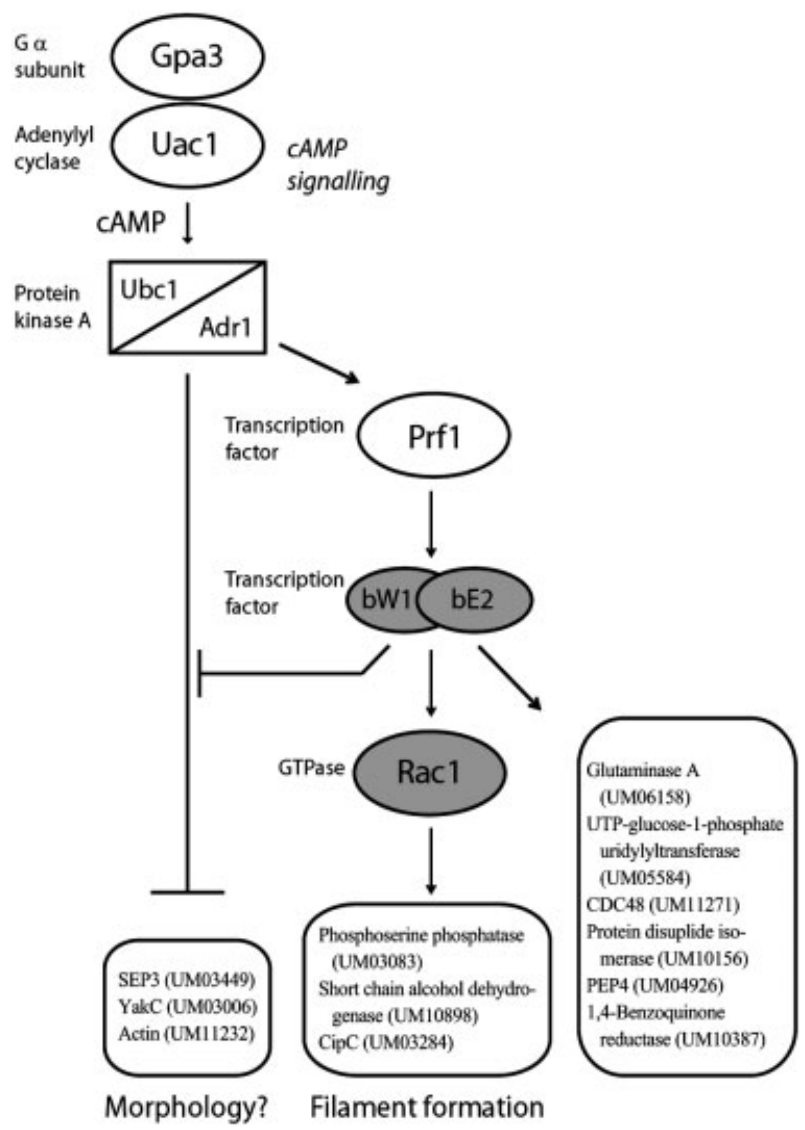

Figure 5. Proposed model of $U$. maydis filamentous growth induction. The cAMP/PKA pathway represses genes, which may be involved in filament morphology, such as SEP3, and transcriptionally activates the $b$-locus. The active b-heterodimer then induces filament formation by a Rac1-containing signalling module and suppresses cAMP/PKA-dependent repression of SEP3, YakC and actin. By the same time the $b$-locus regulates proteins, many of them localized in the secretory compartment, which may have a function in pathogenicity. induced MAPK-pathway and the CAMP signalling pathway $[41,45]$. However, it appears that cAMP signalling does not play a decisive role in the Rac1-dependent pathway of mating type regulated filament formation.

\subsection{Outlook}

Due to the limited number of $U$. maydis proteins represented in our proteome map we clearly could not identify all proteins associated with filamentous growth. A more extensive analysis of the $U$. maydis proteome together with detailed microarray analyses will certainly enhance our understanding of filamentous growth. At the same time such studies will reveal a better understanding of the relation between transcription rate and protein abundance. A comprehensive analysis of mutants defective for the filament-specific proteins identified in this study may provide new insights into the signalling pathways which play a role in the morphogenetic switch from budding to filamentous growth. In addition, the proteins identified in our study may prove to be potential targets for antibiotic substances specifically targeted at dimorphic fungal pathogens.

The authors thank Ursula Wieneke (MS group at the MPIZ Cologne, Germany) for technical assistance. Thomas Colby was supported by the Deutsche Forschungsgemeinschaft, Sonderforschungsbereich (SFB) 635. The project was further funded by grants of the Max-Planck Society to Jürgen Schmidt. Christian Böhmer was supported by the Deutsche Forschungsgemeinschaft, GK 1216.

\section{References}

[1] Bölker, M., Microbiology 2001, 147, 1395-1401.

[2] Feldbrügge, M., Kämper, J., Steinberg, G., Kahmann, R., Curr. Opin. Microbiol. 2004, 7, 666-672.

[3] Kämper, J., Kahmann, R., Bölker, M., Ma, L.-J. et al., Nature 2006, 444. 97-101.

[4] Bölker, M., Urban, M., Kahmann, R., Cell 1992, 68, 441-450.

[5] Kämper, J., Reichmann, M., Romeis, T., Bölker, M., Kahmann, R., Cell 1995, 81, 73-83.

[6] Brachmann, A., Weinzierl, G., Kämper, J., Kahmann, R., Mol. Microbiol. 2001, 42, 1047-1063.

[7] Hall, A., Nobes, C. D., Philos. Trans. R. Soc. Lond., B, Biol. Sci. 2000, 355, 965-970.

[8] Mahlert, M., Leveleki, L., Hlubek, A., Sandrock, B., Bölker, M., Mol. Microbiol. 2006, 59, 567-578.

[9] Scherer, M., Heimel, K., Starke, V., Kamper, J., Plant Cell 2006, 18, 2388-2401.

[10] Banuett, F., Herskowitz, I., PNAS 1989, 86, 5878-5882.

[11] Romeis, T., Brachmann, A., Kahmann, R., Kämper, J., Mol. Microbiol. 2000, 37, 54-66.

[12] Bradford, M. M., Anal. Biochem. 1976, 72, 248-254. 
[13] Gobom, J., Schuerenberg, M., Mueller, M., Theiss, D. et al., Anal. Chem. 2001, 73, 434-438.

[14] Pappin, D. J. C., Hojrup, P., Bleasby, A. J., Curr. Biol. 1993, 3, 327-332.

[15] Emanuelsson, O., Nielsen, H., Brunak, S., von Heijne, G., J. Mol. Biol. 2000, 300, 1005-1016.

[16] Horton, P., Park, K.-J., Obayashi, T., Nakai, K., Proceedings of the 4th Annual Asia Pacific Bioinformatics Conference APBC06, Taipei, Taiwan 2006, pp. 39-48.

[17] Wilson, C. A., Kreychman, J., Gerstein, M., J. Mol. Biol. 2000, 297, 233-249.

[18] Chiocchetti, A., Gibello, L., Carando, A., Aspesi, A. et al., Haematologica 2005, 90, 1453-1462.

[19] Cook, R. K., Sheff, D. R., Rubenstein, P. A., J. Biol. Chem. 1991, 266, 16825-16833.

[20] Andrews, D. L., Garcia-Pedrajas, M. D., Gold, S. E., Mol. Plant Pathol. 2004, 5, 281-293.

[21] Larraya, L. M., Boyce, K. J., So, A., Steen, B. R. et al., Eukaryot. Cell 2005, 4, 2029-2043.

[22] Mori, K., Ogawa, N., Kawahara, T., Yanagi, H., Yura, T., J. Biol. Chem. 1998, 273, 9912-9920.

[23] Gow, N. A. R., Brown, A. J. P., Odds, F. C., Curr. Opin. Microbiol. 2002, 5, 366-371.

[24] Anagnostakis, S. L., Mycologia 1971, 63, 94-97.

[25] Wösten, H. A., Bohlmann, R., Eckerskorn, C., Lottspeich, F. et al., EMBO J. 1996, 15, 4274-4281.

[26] Le Quere, A., Schutzendubel, A., Rajashekar, B., Canback, B. et al., Mol. Ecol. 2004, 13, 3809-3819.

[27] Teichert, S., Schonig, B., Richter, S., Tudzynski, B., Mol. Microbiol. 2004, 53, 1661-1675.

[28] Steen, B. R., Zuyderduyn, S., Toffaletti, D. L., Marra, M. et al., Eukaryot. Cell 2003, 2, 1336-1349.
[29] Sexton, A. C., Cozijnsen, A. J., Keniry, A., Jewell, E. et al., FEMS Microbiol. Lett. 2006, 258, 150-160.

[30] Snoeijers, S. S., Pérez-García, A., Joosten, M. H. A. J., de Wit, P. J. G. M., Eur. J. Plant Pathol. 2000, 106, 493-506.

[31] Coleman, M., Henricot, B., Arnau, J., Oliver, R. P., Mol. Plant Microbe Interact. 1997, 10, 1106-1109.

[32] Talbot, N. J., Ebbole, D. J., Hamer, J. E., Plant Cell 1993, 5, 1575-1590.

[33] Stephenson, S. A., Green, J. R., Manners, J. M., Maclean, D. J., Curr. Genet. 1997, 31, 447-454.

[34] Gimeno, C. J., Ljungdahl, P. O., Styles, C. A., Fink, G. R., Cell 1992, 68, 1077-1090.

[35] Regenfelder, E., Spellig, T., Hartmann, A., Lauenstein, S. et al., EMBO J. 1997, 16, 1934-1942.

[36] Boyce, K. J., Chang, H., D'Souza, C. A., Kronstad, J. W., Eukaryot. Cell 2005, 4, 2044-2056.

[37] Gold, S., Duncan, G., Barrett, K., Kronstad, J., Genes Dev. 1994, 8, 2805-2816.

[38] Orth, A. B., Rzhetskaya, M., Pell, E. J., Tien, M., Appl. Environ. Microbiol. 1995, 61, 2341-2345.

[39] Durrenberger, F., Wong, K., Kronstad, J. W., Proc. Natl. Acad. Sci. USA 1998, 95, 5684-5689.

[40] Loubradou, G., Brachmann, A., Feldbrugge, M., Kahmann, R., Mol. Microbiol. 2001, 40, 719-730.

[41] Kaffarnik, F., Muller, P., Leibundgut, M., Kahmann, R., Feldbrugge, M., EMBO J. 2003, 22, 5817-5826.

[42] Urban, M., Kahmann, R., Bolker, M., Mol. Gen. Genet 1996, 251, 31-37.

[43] Longtine, M. S., Bi, E., Trends Cell Biol. 2003, 13, 403-409.

[44] D'Souza, C. A., Heitman, J., FEMS Microbiol. Rev. 2001, 25, 349-364.

[45] Kruger, J., Loubradou, G., Regenfelder, E., Hartmann, A., Kahmann, R., Mol. Gen. Genet. 1998, 260, 193-198. 